\title{
The impact of triglyceride-glucose index on incident cardiovascular events during 16 years of follow-up: Tehran Lipid and Glucose Study
}

\author{
Niloofar Barzegar ${ }^{1}$, Maryam Tohidi ${ }^{*}{ }^{*}$, Mitra Hasheminia ${ }^{1}$, Fereidoun Azizi ${ }^{2}$ and Farzad Hadaegh ${ }^{1}$
}

\begin{abstract}
Background: To investigate whether the Triglyceride-Glucose index (TyG-index) is associated with increased risk of cardiovascular diseases (CVD)/coronary heart disease (CHD).

Methods: A total of 7521 Iranians aged $\geq 30$ years (male $=3367$ ) were included in the study. Multivariate Cox regression analyses (adjusted for age, gender, waist circumference, body mass index, educational level, smoking status, physical activity, family history of CVD, type 2 diabetes, hypertension, low and high density lipoprotein cholesterol, and lipid lowering drugs) were used to assess the risk of incident CVD/CHD across quintiles and for 1-standard deviation (SD) increase in the TyG-index. The cut off point for TyG-index was assessed by the minimum value of $\sqrt{(1-\text { sensitivity })^{2}+(1-\text { specificity })^{2}}$. We also examined the added value of the TyG-index in addition to the Framingham risk score when predicting CVD.

Results: During follow-up, 1084 cases of CVD (male $=634$ ) were recorded. We found a significant trend of TyG-index for incident CVD/CHD in multivariate analysis (both Ps for tend $\leq 0.002$ ). Moreover, a 1-SD increase in TyG-index was associated with significant risk of CVD/CHD in multivariate analysis [1.16 (1.07-1.25) and 1.19 (1.10-1.29), respectively]. The cut-off value of TyG-index for incident CVD was 9.03 (59.2\% sensitivity and 63.2\% specificity); the corresponding value of TyG-index for incident CHD was 9.03 (60.0\% sensitivity and 62.8\% specificity), respectively. Although no interaction was found between gender and TyG-index for CVD/CHD in multivariate analysis (both Ps for interaction > 0.085), the significant trend of TyG-index was observed only among females for incident CVD $(P=0.035)$. A significant interaction was found between age groups (i.e. $\geq 60$ vs $<60$ years) and TyG-index for CVD outcomes in the multivariate model (P-value for interaction =0.046). Accordingly, a significant association between the TyG-index and outcomes was found only among the younger age group. Among the population aged $<60$ the addition of TyG-index to the Framingham risk score (FRS) did not show improvement in the predictive ability of the FRS, using integrated discrimination improvement.

Conclusion: The TyG-index is significantly associated with increased risk of CVD/CHD incidence; this issue was more prominent among the younger population. However, adding TyG-index to FRS does not provide better risk prediction for CVD.
\end{abstract}

\footnotetext{
*Correspondence: tohidi@endocrine.ac.ir

1 Prevention of Metabolic Disorders Research Center, Research Institute for Endocrine Sciences, Shahid Beheshti University of Medical Sciences, No. 24 Aarabi St. Velenjak, P.O. Box: 19395-4763, Tehran, Iran

Full list of author information is available at the end of the article
}

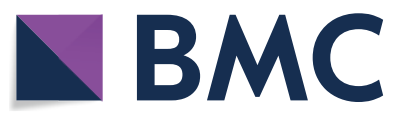

(c) The Author(s) 2020. This article is licensed under a Creative Commons Attribution 4.0 International License, which permits use, sharing, adaptation, distribution and reproduction in any medium or format, as long as you give appropriate credit to the original author(s) and the source, provide a link to the Creative Commons licence, and indicate if changes were made. The images or other third party material in this article are included in the article's Creative Commons licence, unless indicated otherwise in a credit line to the material. If material is not included in the article's Creative Commons licence and your intended use is not permitted by statutory regulation or exceeds the permitted use, you will need to obtain permission directly from the copyright holder. To view a copy of this licence, visit http://creativeco mmons.org/licenses/by/4.0/. The Creative Commons Public Domain Dedication waiver (http://creativecommons.org/publicdomain/ zero/1.0/) applies to the data made available in this article, unless otherwise stated in a credit line to the data. 
Keywords: Cardiovascular disease, Coronary heart disease, TyG-index

\section{Background}

Over the past several years, cardiovascular diseases (CVD), including ischemic heart disease, heart failure, stroke, and several other cardiac and vascular conditions have played an important role in the global rate of mortality [1]. As with the rest of the world, CVD is the primary cause of morbidity and mortality in the Middle East and North Africa (MENA) [2]. It has been reported that about $80 \%$ of global CVD mortality is attributable to middle- and low-income countries where the burden of CVD and CVD risk factors are on the rise [3]. This fact makes it vital to recognize and control potential risk factors.

Insulin resistance (IR) is known to be one of the most important risk factors for CVD [4]. Not only is IR associated with CVD risk factors such as type 2 diabetes (T2D), hypertension, dyslipidemia, and obesity, but it is also an independent risk factor for CVD [5]. In this regard, Triglyceride-Glucose index (TyG-index), a product of triglyceride (TG) and fasting plasma glucose (FPG), is evaluated as a surrogate for IR [6-8]. Some studies have shown an association between TyG-index and incident T2D [9-12]. Cohort studies conducted among American, European, and Chinese populations have found increasing TyG-index level as an independent risk factor for incident CVD [13-17].

Due to the high burden of IR and CVD among residents of the MENA region [18], we examined the association between TyG-index and incident CVD and coronary heart disease (CHD) for the first time, during a follow-up of more than a decade in the oldest cohort of the region namely the Tehran Lipid and Glucose Study (TLGS). Moreover, we also examined these associations in each gender, and among elderly vs younger adult populations. Appropriate cut off points were also derived for this index in prediction of CVD/CHD events. Finally, the added value of TyG-index when included in the Framingham risk score (FRS) [19] in prediction of CVD was also examined.

\section{Methods}

\section{Study population}

This study was conducted within the framework of TLGS. TLGS is an ongoing large-scale community-based cohort study with a long-term follow-up. The study was initiated in 1999-2002 to estimate the prevalence of noncommunicable diseases and related risk factors. Detailed descriptions and study design of TLGS have been reported elsewhere [20].
In brief, TLGS includes 15,005 participants at first visit (1999-2002), with an additional 3550 recruitments in the second visit (2002-2005). Follow-up visits are performed at approximately 3-year intervals. Participants of the TLGS aged $\geq 30$ years $(n=9554)$ were included in this study (7928 from the first phase and 1626 from the second phase). After exclusion of subjects with prevalent CVD at baseline $(n=602)$, those with missing data for covariates including anthropometric measures, smoking status, T2D, hypertension, family history of CVD, and lipid measures $(n=749$, considering overlap features between numbers), and finally those without any followup after baseline recruitment $(n=682), 7521$ individuals remained in the study and were followed until March 2016.

This study was approved by the Ethics Committee of the Research Institute for Endocrine Science, Shahid Beheshti University of Medical Sciences, and all participants provided written informed consent.

\section{Clinical, anthropometric, and laboratory measurements}

Participants were interviewed individually by trained interviewers. A standard pre-tested questionnaire was used to collect demographic information. Subjects were questioned about their physical activity, smoking status, level of education, drug history, past medical history, and family history. During the first phase of TLGS, physical activity pattern was assessed by the Lipid Research Clinic (LRC) questionnaire. As a result of the insufficient accuracy of LRC [21], it was replaced by the Modifiable Activity Questionnaire (MAQ) for future follow-up examinations [22]. Details of anthropometric measurements including weight, height, and waist circumference (WC) are reported elsewhere [20]. Body mass index (BMI) was calculated by dividing weight $(\mathrm{kg})$ by squared height $\left(\mathrm{m}^{2}\right)$. After $15 \mathrm{~min}$ rest in a sitting position, a physician measured blood pressure using a calibrated mercury sphygmomanometer. Systolic blood pressure (SBP) and diastolic blood pressure (DBP) were measured twice with at least $30 \mathrm{~min}$ interval and the mean value was considered as the participant's blood pressure.

Blood samples were drawn between 7:00 and 9:00 am after 12 to $14 \mathrm{~h}$ of overnight fasting. Samples were centrifuged within 30-45 min of collection. Details of laboratory measures including total cholesterol (TC), TG, high-density lipoprotein cholesterol (HDL-C), FPG, and $2 \mathrm{~h}$ plasma glucose (2hPG) are reported elsewhere $[20,23]$. Low-density lipoprotein cholesterol (LDLC) was calculated from serum TC, HDL-C, and TG 
concentrations expressed in $(\mathrm{mg} / \mathrm{dL})$ using the Friedewald formula [24].

\section{Assessment and definition of the outcome}

Follow-up for any medical outcome was repeated annually by phone call by a trained nurse. For medical conditions leading to hospitalization or mortality, a trained physician collected complementary data through a home visit and hospital documents [25]. Collected data were evaluated by an outcome committee consisting of an internist, endocrinologist, cardiologist, epidemiologist, pathologist and other experts when needed, in order to assign a specific outcome to every event. It is important to note that the outcome committee was blinded to the status of baseline risk factors.

In the current study, CHD was defined as any definite myocardial infarction (MI) diagnosed by electrocardiogram (ECG) and biomarkers (including troponin CK, CK-MB, CK-MBm, and myoglobin), probable MI (positive ECG findings plus cardiac symptoms and missing biomarkers or positive ECG findings plus equivocal biomarkers), unstable angina pectoris (new cardiac symptoms or changes in the symptom pattern, and positive ECG findings with normal biomarker values), and angiography proven CHD. CVD was determined as a composite of CHD plus fatal and non-fatal stroke.

\section{Definition of terms}

The TyG-index was calculated as $\ln$ [fasting TG $(\mathrm{mg} /$ $\mathrm{dL}) \times$ FPG $(\mathrm{mg} / \mathrm{dL}) / 2$ ] [26]. The categorization of the TyG-index among the total population was as follows: quintile $1(<8.4)$ (as reference), quintile 2 (8.4-8.7), quintile 3 (8.7-9.0), quintile $4(9.0-9.4)$, and quintile 5 (>9.4). Hypertension was specified as $\mathrm{SBP} \geq 140 \mathrm{mmHg}$ or $\mathrm{DBP} \geq 90 \mathrm{mmHg}$ or the use of antihypertensive drugs. T2D was ascertained among participants who had FPG $\geq 7 \mathrm{mmol} / \mathrm{L}, 2 \mathrm{hPG} \geq 11.1 \mathrm{mmol} / \mathrm{L}$, or current use of anti-diabetic medication. We divided educational status into three groups: illiterate and $<6$ years of education (as reference), 6-12 years, and $\geq 12$ years which can be considered as primary, secondary and superior levels of education, respectively. Low physical activity was defined as exercising less than 3 days per week (according to LRC questionnaire) [21] and scores $\leq 600$ MET (metabolic equivalent task)-minutes per week (according to MAQ questionnaire) [27] for participants who entered the study at the first and second phases of TLGS, respectively. A current smoker was defined as a person who smokes cigarettes, pipe or hookah daily or occasionally; those who were not currently smokers with a previous history of smoking were called past smokers; and those without any history of smoking were called never smokers (as a reference group).

\section{Statistical analysis}

Continuous variables were expressed as the mean \pm standard deviation (SD). Categorical variables are presented as frequency and percentage. We used one-way ANOVA, and Chi-squared test to compare the baseline characteristics of subgroups across TyG-index quintiles.

The cumulative incidence of CVD/CHD were calculated by dividing the number of cases by the number of subjects followed for each TyG-index category.

Since there were no interactions between gender and TyG-index for CVD/CHD in multivariate analysis (both Ps for interaction $>0.085$ ), the analysis was performed among the entire population. However, to be comparable with other studies, we also performed data analysis in each gender. Multivariate Cox proportionalhazard analyses were performed to estimate hazard ratios (HRs) and 95\% confidence intervals (CIs) of incident CVD/CHD. TyG-index was examined both across quintiles and for a 1-standard deviation (SD) increase. All analyses were adjusted for potential CVD/CHD confounders including age, gender (for total population), WC, BMI, educational level, smoking status, physical activity, family history of CVD, T2D, hypertension, LDL-C, HDL-C, and lipid lowering drugs based on biological plausibility and identification as a confounder in our previous studies [28]. We examined the variance inflation factor (VIF) of the variables included in the model to address the issue of collinearity [29]. We did not find evidence of collinearity in the model, given the VIF of $<5$.

To determine the optimum cut off value for TyG-index in cases of incident CVD/CHD, we used the receiver operating characteristic (ROC) curve analysis. The best cut off point for TyG-index was assessed by the minimum value of $\sqrt{(1-\text { sensitivity })^{2}+(1-\text { specificity })^{2}}$, which represented the maximum sum of sensitivity and specificity.

Since we found significant interaction between age groups (i.e. $\geq 60$ vs $<60$ years) and TyG-index for CVD outcomes in the multivariate model ( $\mathrm{P}$ for interaction $=0.046$ ), we performed analysis according to age groups.

Finally, we fitted a prediction model based on variables used in the FRS including age, gender, TC, HDL-C, SBP, anti-hypertensive drugs, smoking, and T2D. Afterwards we developed a new model comprising the Framingham variables with the addition of baseline TyG-index. In this regard, we used Harrell's concordance statistic (C-index) 
to evaluate discrimination of the predicting models (FRS model with and without TyG-index) and integrated discrimination improvement (IDI) as a criterion for evaluating the ability of TyG-index to improve CVD risk prediction over FRS [30, 31].

The proportional hazard assumption in the Cox models was assessed with the Schoenfild residual test; all proportionality assumptions were appropriate. Statistical analysis was performed using SPSS for windows version 20 and STATA version 14 . A P-value of $\leq 0.05$ was considered to indicate statistical significance.

\section{Results}

\section{Baseline characteristics}

Overall, a total of 7521 subjects (male $=3367$ ) were included in the analysis. Baseline characteristics of the study population according to TyG-index quintiles are presented in Table 1. Generally, compared to the lowest quintile of TyG-index, those in the highest quintile were older, less likely to be female, had higher values of WC and BMI, were less educated and less physically active.
Moreover, the prevalence of T2D, hypertension, and the use of lipid lowering drugs as well as baseline values of LDL-C were higher, whereas the value of HDL-C was lower among participants in the fifth quintile of the TyG-index.

After a median follow-up of 16.1 years (interquartile range: 13.4-16.5), $1084 \quad($ male $=634)$ and 924 (male $=542$ ) cases of CVD and CHD occurred.

\section{TyG-index as a categorical variable}

According to Fig. 1, changes in TyG-index levels from the first to fifth quintiles showed significant increasing trends $(\mathrm{P}$-value $=0.002$ and $<0.001)$ for incident CVD and $\mathrm{CHD}$, respectively. Compared to the reference, adjusted HRs (95\% CI) for incident CVD for the second, third, fourth and fifth quintiles were $1.15(0.89-1.49)$, 1.28 (0.99-1.65), $1.22(0.94-1.58)$, and 1.61 (1.23-2.11); and the corresponding HRs for incident CHD were 1.25 (0.93-1.67), 1.49 (1.12-1.98), 1.34 (1.01-1.80), and $1.84(1.37-2.48)$, respectively. According to multivariate Cox regression analysis for incident CVD, aging, abdominal adiposity, smoking, family history of

Table 1 Baseline characteristics of the study population by quintiles of TyG-index: Tehran Lipid and Glucose Study

\begin{tabular}{|c|c|c|c|c|c|c|c|}
\hline \multirow[t]{2}{*}{ Variables } & \multicolumn{5}{|c|}{ TyG-index quartiles } & \multirow[t]{2}{*}{ P-value } & \multirow[t]{2}{*}{ Total $(n=7521)$} \\
\hline & $\begin{array}{l}1(<8.4) \\
(n=1503)\end{array}$ & $\begin{array}{l}2(8.4-8.7) \\
(n=1506)\end{array}$ & $\begin{array}{l}3(8.7-9.0) \\
(n=1504)\end{array}$ & $\begin{array}{l}4(9.0-9.4) \\
(n=1504)\end{array}$ & $\begin{array}{l}5(\geq 9.4) \\
(n=1504)\end{array}$ & & \\
\hline Age (years) & $43.1 \pm 12.3$ & $45.5 \pm 12.2$ & $47.1 \pm 12.0$ & $47.3 \pm 11.7$ & $50.3 \pm 11.4$ & $<0.001$ & $46.6 \pm 12.1$ \\
\hline \multicolumn{8}{|l|}{ Gender } \\
\hline Male & $588(39.1)$ & $664(44.1)$ & $696(46.3)$ & $696(46.3)$ & $723(48.1)$ & \multirow[t]{2}{*}{$<0.001$} & 3367 (44.8) \\
\hline Female & $915(60.9)$ & $842(55.9)$ & $808(53.7)$ & $808(53.7)$ & $781(51.9)$ & & $4154(55.2)$ \\
\hline $\mathrm{WC}(\mathrm{cm})$ & $83.2 \pm 10.7$ & $88.3 \pm 10.9$ & $91.7 \pm 10.3$ & $94.0 \pm 10.6$ & $96.5 \pm 10.2$ & $<0.001$ & $90.7 \pm 11.5$ \\
\hline $\mathrm{BMI}\left(\mathrm{kg} / \mathrm{m}^{2}\right)$ & $25.1 \pm 4.27$ & $26.8 \pm 4.39$ & $27.9 \pm 4.40$ & $28.7 \pm 4.32$ & $29.1 \pm 4.32$ & $<0.001$ & $27.5 \pm 4.57$ \\
\hline \multicolumn{8}{|l|}{ Education (years) } \\
\hline$<6$ & $451(30.0)$ & $560(37.2)$ & $604(40.2)$ & $639(42.5)$ & $789(52.5)$ & \multirow[t]{3}{*}{$<0.001$} & $3043(40.5)$ \\
\hline $6-12$ & $834(55.5)$ & $749(49.7)$ & $708(47.1)$ & $698(46.4)$ & $559(37.2)$ & & $3548(47.2)$ \\
\hline$\geq 12$ & $218(14.5)$ & $197(13.1)$ & $192(12.8)$ & $167(11.1)$ & $156(10.4)$ & & $930(12.4)$ \\
\hline \multicolumn{8}{|l|}{ Smoking } \\
\hline Never & $1164(77.4)$ & $1118(74.2)$ & $1134(75.4)$ & $1118(74.3)$ & $1101(73.2)$ & \multirow[t]{3}{*}{0.075} & $5635(74.9)$ \\
\hline Past & $104(6.9)$ & $114(7.6)$ & $122(8.1)$ & $140(9.3)$ & $142(9.4)$ & & $622(8.3)$ \\
\hline Current & 235 (15.6) & $274(18.2)$ & $248(16.5)$ & $246(16.4)$ & $261(17.4)$ & & $1264(16.8)$ \\
\hline Low physical activity & $1002(66.7)$ & $996(66.1)$ & 1066 (70.9) & $1056(70.2)$ & $1071(71.2)$ & 0.002 & $5191(69.0)$ \\
\hline $\mathrm{FH}-\mathrm{CVD}$ & $227(15.1)$ & 239(15.9) & $239(15.9)$ & $253(16.8)$ & $264(17.6)$ & 0.407 & $1222(16.2)$ \\
\hline $\mathrm{T} 2 \mathrm{D}$ & $16(1.1)$ & $54(3.6)$ & $78(5.2)$ & $195(13.0)$ & $645(42.9)$ & $<0.001$ & $988(13.1)$ \\
\hline Hypertension & $162(10.8)$ & $281(18.7)$ & $375(24.9)$ & 464 (30.9) & $577(38.4)$ & $<0.001$ & $1859(24.7)$ \\
\hline Lipid Lowering drugs & $6(0.4)$ & $18(1.2)$ & $41(2.7)$ & $54(3.6)$ & $154(10.2)$ & $<0.001$ & $273(3.6)$ \\
\hline LDL-C (mmol/L) & $2.98 \pm 0.76$ & $3.40 \pm 0.81$ & $3.61 \pm 0.83$ & $3.76 \pm 0.92$ & $3.91 \pm 1.06$ & $<0.001$ & $3.53 \pm 0.94$ \\
\hline $\mathrm{HDL}-\mathrm{C}(\mathrm{mmol} / \mathrm{L})$ & $1.23 \pm 0.29$ & $1.13 \pm 0.29$ & $1.05 \pm 0.26$ & $1.01 \pm 0.24$ & $0.95 \pm 0.25$ & $<0.001$ & $1.07 \pm 0.28$ \\
\hline
\end{tabular}

Data are represented as mean \pm SD or frequency (percent)

TyG-index triglyceride and glucose index, WC waist circumference, $B M I$ body mass index, $F H$-CVD family history of cardiovascular disease, $T 2 D$ type 2 diabetes, $L D L-C$ low-density lipoprotein cholesterol, $H D L-C$ high-density lipoprotein cholesterol 


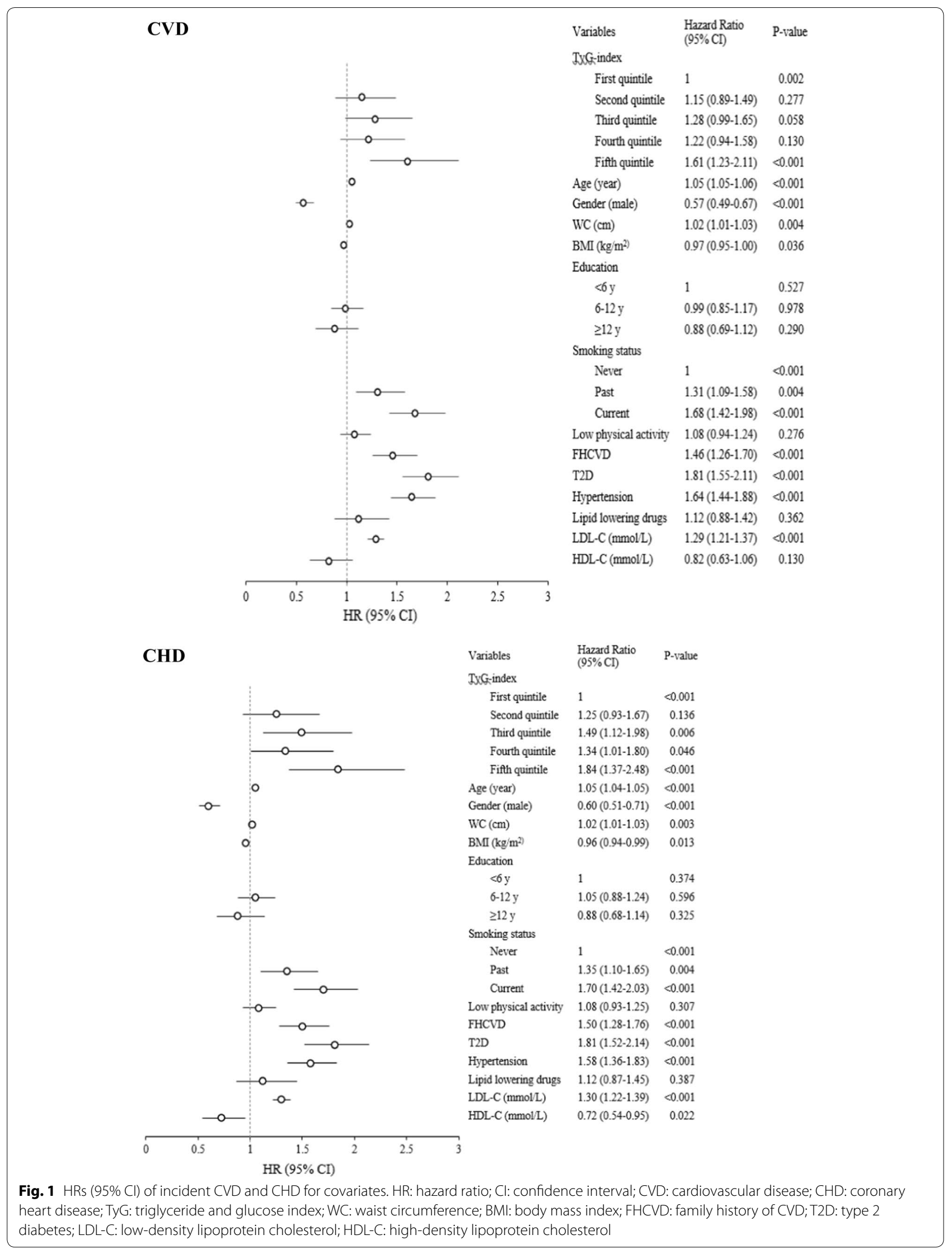


CVD, T2D, hypertension, and a higher value of LDL-C increased the risk of CVD events; whereas, BMI and female gender were associated with lower risk. Similar results are shown in Fig. 1 for incident CHD; however, a significant association was found between higher values of HLD-C and lower risk for incident CHD. Regarding the inverse association of $\mathrm{BMI}$ for incident CVD/CHD in our data analysis, after excluding $\mathrm{WC}$ and obesity mediators (including T2D, hypertension, LDL-C, HDL$\mathrm{C}$, and TyG-index) [32], higher BMI was significantly associated with higher risk of CVD/CHD events [HR: 1.04, 95\% CI (1.03-1.06)]; the risks however, reached the null after further adjustment for obesity mediators. After further adjustment for WC, higher BMI was associated with lower risk of events (Additional file 1: Table S1 and S2).

\section{TyG-index as a continuous variable}

Table 2 shows the association between 1SD increase in TyG-index and incident CVD based on multivariate Cox proportional hazard analyses among males, females and total population. Accordingly, 1SD increase in TyG-index for incident CVD were 1.11 (1.01-1.22),
1.23 (1.09-1.39), and 1.16 (1.07-1.25), respectively; the corresponding values for incident CHD were 1.16 (1.05-1.29), 1.23 (1.07-1.39), and 1.19 (1.10-1.29), (Table 3).

\section{TyG-index cut off points}

Among population with complete follow-up $(n=6463)$, area under curve (AUC) of the TyG-index for incidence CVD/CHD were $0.633,95 \%$ CI $(0.645-0.681)$ and 0.669 , 95\% CI (0.651-0.688), respectively (Fig. 2). The cut-off points for TyG-index for incident CVD/CHD among the whole population are also shown in Fig. 2. Cut-off value for TyG-index for incident CVD was calculated to be 9.03 with $59.23 \%$ sensitivity and $63.15 \%$ specificity; the corresponding values incident CHD was also 9.03 with 59.96\% sensitivity and $62.84 \%$ specificity, respectively.

\section{Sensitivity analysis}

To assess the robustness of our findings, we also performed sensitivity analyses according to gender, and among age groups, separately, in the multivariate adjusted models (Fig. 3). Although no interaction was found between gender and TyG-index for CVD/CHD in multivariate analysis (Ps for interaction $>0.085$ ), the

Table 2 Multivariable-adjusted hazard ratios of incident CVD according to the 1-SD increase in TyG-index, stratified by gender: Tehran Lipid and Glucose Study

\begin{tabular}{|c|c|c|c|c|c|c|}
\hline \multirow[t]{2}{*}{ Variables } & \multicolumn{2}{|l|}{ Male $(n=4154)$} & \multicolumn{2}{|c|}{ Female $(n=3367)$} & \multicolumn{2}{|l|}{ Total $(n=7521)$} \\
\hline & $\mathrm{HR}(95 \% \mathrm{Cl})$ & P value & $\mathrm{HR}(95 \% \mathrm{CI})$ & $P$ value & $\mathrm{HR}(95 \% \mathrm{Cl})$ & P value \\
\hline TyG-index & $1.11(1.01-1.22)$ & 0.040 & $1.23(1.09-1.39)$ & 0.001 & $1.16(1.07-1.25)$ & $<0.001$ \\
\hline Age (years) & $1.06(1.05-1.06)$ & $<0.001$ & 1.05 (1.04-1.06) & $<0.001$ & $1.05(1.05-1.06)$ & $<0.001$ \\
\hline WC $(\mathrm{cm})$ & $1.01(1.00-1.03)$ & 0.105 & $1.02(1.00-1.03)$ & 0.022 & $1.02(1.01-1.03)$ & 0.004 \\
\hline BMI $\left(\mathrm{kg} / \mathrm{m}^{2}\right)$ & $0.98(0.93-1.02)$ & 0.261 & $0.98(0.94-1.01)$ & 0.155 & $0.97(0.95-1.00)$ & 0.052 \\
\hline \multicolumn{7}{|l|}{ Education (years) } \\
\hline$<6$ & Reference & & Reference & & Reference & \\
\hline $6-12$ & $1.11(0.92-1.35)$ & 0.289 & $0.81(0.61-1.07)$ & 0.135 & $0.99(0.85-1.16)$ & 0.922 \\
\hline$\geq 12$ & $0.98(0.75-1.29)$ & 0.903 & $0.59(0.30-1.16)$ & 0.124 & $0.88(0.69-1.12)$ & 0.299 \\
\hline \multicolumn{7}{|l|}{ Smoking } \\
\hline Never & Reference & & Reference & & Reference & \\
\hline Past & $1.37(1.12-1.69)$ & 0.003 & $1.01(0.63-1.61)$ & 0.983 & $1.31(1.09-1.57)$ & 0.005 \\
\hline Current & $1.67(1.39-2.02)$ & $<0.001$ & $1.62(1.07-2.46)$ & 0.023 & $1.67(1.41-1.97)$ & $<0.001$ \\
\hline Low physical activity & $1.05(0.88-1.25)$ & 0.608 & $1.11(0.90-1.38)$ & 0.333 & $1.07(0.94-1.23)$ & 0.301 \\
\hline FH-CVD & $1.37(1.10-1.70)$ & 0.004 & $1.58(1.28-1.96)$ & $<0.001$ & $1.47(1.26-1.71)$ & $<0.001$ \\
\hline $\mathrm{T} 2 \mathrm{D}$ & $1.73(1.39-2.15)$ & $<0.001$ & $1.80(1.41-2.30)$ & $<0.001$ & $1.78(1.51-2.09)$ & $<0.001$ \\
\hline Hypertension & $1.60(1.34-1.91)$ & $<0.001$ & $1.65(1.34-2.04)$ & $<0.001$ & $1.64(1.43-1.87)$ & $<0.001$ \\
\hline Lipid drug & $0.97(0.63-1.50)$ & 0.895 & $1.20(0.90-1.61)$ & 0.208 & $1.12(0.88-1.42)$ & 0.346 \\
\hline LDL-C (mmol/L) & $1.37(1.25-1.49)$ & $<0.001$ & $1.18(1.07-1.29)$ & 0.001 & $1.28(1.21-1.37)$ & $<0.001$ \\
\hline $\mathrm{HDL}-\mathrm{C}(\mathrm{mmol} / \mathrm{L})$ & $0.76(0.53-1.10)$ & 0.146 & $0.87(0.60-1.25)$ & 0.439 & $0.82(0.63-1.06)$ & 0.133 \\
\hline Gender (female) & - & - & - & - & $0.57(0.49-0.66)$ & $<0.001$ \\
\hline
\end{tabular}


Table 3 Multivariable-adjusted hazard ratios of incident CHD according to the 1-SD increase in TyG-index, stratified by genders: Tehran Lipid and Glucose Study

\begin{tabular}{|c|c|c|c|c|c|c|}
\hline \multirow[t]{2}{*}{ Variables } & \multicolumn{2}{|l|}{ Male $(n=4154)$} & \multicolumn{2}{|c|}{ Female $(n=3366)$} & \multicolumn{2}{|l|}{ Total $(n=7520)$} \\
\hline & $\mathrm{HR}(95 \% \mathrm{Cl})$ & $P$ value & $\mathrm{HR}(95 \% \mathrm{Cl})$ & $P$ value & $\mathrm{HR}(95 \% \mathrm{Cl})$ & $P$ value \\
\hline TyG-index & $1.16(1.05-1.29)$ & 0.005 & $1.23(1.07-1.39)$ & 0.003 & $1.19(1.10-1.29)$ & $<0.001$ \\
\hline Age (years) & $1.05(1.04-1.06)$ & $<0.001$ & $1.04(1.03-1.05)$ & $<0.001$ & $1.05(1.04-1.05)$ & $<0.001$ \\
\hline$W C(\mathrm{~cm})$ & $1.02(1.00-1.04)$ & 0.036 & $1.02(1.00-1.03)$ & 0.066 & $1.02(1.01-1.03)$ & 0.004 \\
\hline $\mathrm{BMI}\left(\mathrm{kg} / \mathrm{m}^{2}\right)$ & $0.96(0.92-1.01)$ & 0.100 & $0.97(0.94-1.01)$ & 0.162 & $0.97(0.94-1.00)$ & 0.020 \\
\hline \multicolumn{7}{|l|}{ Education (years) } \\
\hline$<6$ & Reference & & Reference & & Reference & \\
\hline $6-12$ & $1.21(0.98-1.49)$ & 0.075 & $0.81(0.60-1.09)$ & 0.161 & $1.04(0.88-1.23)$ & 0.629 \\
\hline$\geq 12$ & $1.02(0.76-1.36)$ & 0.904 & $0.57(0.27-1.17)$ & 0.122 & $0.88(0.68-1.15)$ & 0.343 \\
\hline \multicolumn{7}{|l|}{ Smoking } \\
\hline Never & Reference & & Reference & & Reference & \\
\hline Past & $1.39(1.11-1.75)$ & 0.004 & $1.08(0.65-1.80)$ & 0.768 & $1.34(1.09-1.64)$ & 0.005 \\
\hline Current & $1.67(1.37-2.04)$ & $<0.001$ & $1.68(1.08-2.59)$ & 0.020 & $1.68(1.41-2.01)$ & $<0.001$ \\
\hline Low physical activity & $1.09(0.90-1.32)$ & 0.382 & $1.06(0.84-1.33)$ & 0.650 & $1.08(0.93-1.25)$ & 0.325 \\
\hline FH-CVD & $1.39(1.10-1.74)$ & 0.005 & $1.63(1.30-2.06)$ & $<0.001$ & $1.51(1.28-1.77)$ & $<0.001$ \\
\hline $\mathrm{T} 2 \mathrm{D}$ & $1.53(1.20-1.95)$ & 0.001 & $2.01(1.54-2.61)$ & $<0.001$ & $1.74(1.46-2.08)$ & $<0.001$ \\
\hline Hypertension & $1.45(1.19-1.76)$ & $<0.001$ & $1.73(1.37-2.17)$ & $<0.001$ & $1.57(1.35-1.81)$ & $<0.001$ \\
\hline Lipid drug & $0.91(0.56-1.48)$ & 0.696 & $1.24(0.91-1.69)$ & 0.171 & $1.12(0.87-1.45)$ & 0.380 \\
\hline LDL-C (mmol/L) & $1.37(1.25-1.50)$ & $<0.001$ & $1.21(1.09-1.33)$ & $<0.001$ & $1.30(1.22-1.39)$ & $<0.001$ \\
\hline $\mathrm{HDL}-\mathrm{C}(\mathrm{mmol} / \mathrm{L})$ & $0.71(0.48-1.06)$ & 0.097 & $0.69(0.46-1.03)$ & 0.072 & $0.72(0.54-0.96)$ & 0.023 \\
\hline Gender (female) & - & - & - & - & $0.60(0.50-0.71)$ & $<0.001$ \\
\hline
\end{tabular}

CHD coronary heart disease, SD standard deviation, TyG-index triglyceride glucose-index, $H R$ hazard ratio, $C I$ confidence interval, WC waist circumference, $B M I$ body mass index, FH-CVD family history of cardiovascular disease, T2D type 2 diabetes, LDL-C low density lipoprotein cholesterol, HDL-C high density lipoprotein cholesterol

significant trend of TyG-index was observed only among females for incident CVD $(\mathrm{P}=0.035)$. HRs and 95\% CIs from the second to fifth quintile were $1.69(1.01-2.81)$, 1.74 (1.05-2.87), 1.77 (1.07-2.92), and 2.25 (1.34-3.77), respectively. The corresponding values for incident $\mathrm{CHD}$ were 1.54 (0.88-2.70), 1.83 (1.07-3.14), 1.64 (0.95-2.83), and 2.09 (1.19-3.65), respectively.

Age-stratified analyses showed a significant trend of TyG-index (Ps for trend $\leq 0.010$ ) for incident CVD/CHD among the younger age group ( $<60$ years); however, risks reached the null in subjects who were 60 years or older. The adjusted HR of TyG-index from the second to fifth quintile, among population aged $<60$ years for incident CVD were 1.41 (0.98-2.01), 1.52 (1.07-2.16), 1.61 (1.132.29), 1.96 (1.36-2.83)] corresponding values for incident CHD were 1.33 (0.91-1.93), 1.57 (1.09-2.27), 1.56 (1.082.25), 1.94 (1.33-2.83), respectively (Fig. 3).

\section{Added value of TyG-index to FRS}

The predictive value of TyG-index for CVD events was assessed using Framingham risk score components among population aged $<60$ years. As shown in Table 4, in the model including both FRS and TyG-index, the HR of these variables for incident CVD was 1.06 (1.05-1.06) and 1.42 (1.26-1.60), respectively. In addition, the $\mathrm{C}$-index of the FRS model did not change after addition of the TyG-index [0.79 (0.77-0.81) and 0.77 (0.75-0.79), respectively]. IDI statistics did not show any improvement in the predictive ability of the FRS model [0.001 -0.004 to 0.007$)]$.

\section{Discussion}

In the present study, we found that higher plasma TyGindex level, either as a continuous or categorical variable was associated with increased risk for $\mathrm{CVD} / \mathrm{CHD}$ incidence, independent of other traditional CVD risk factors. The TyG-index cut-off values for both incident CVD and CHD were the same (9.03) with sensitivity and specificity of around $60 \%$. Moreover, a significant association between TyG-index and CVD/CHD was mainly observed among younger adults; although no improvement was observed in prediction of CVD beyond that achieved by FRS.

TyG-index was previously introduced as a surrogate marker of IR [6]. Previous studies have reported an association between IR and important CVD risk factors such as T2D and hypertension [33, 34]. IR is also considered to be strongly correlated with the risk of incident CVD [35, 


\section{CVD}

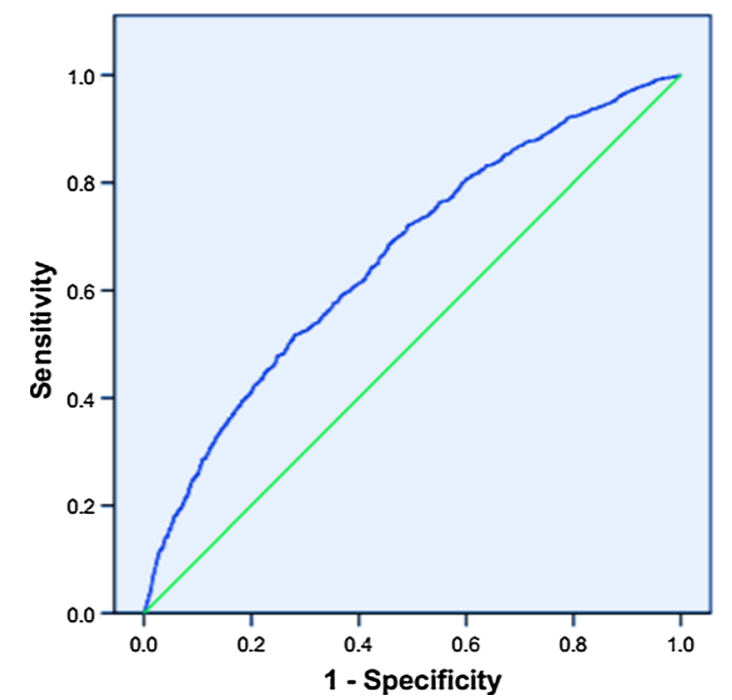

\section{CHD}

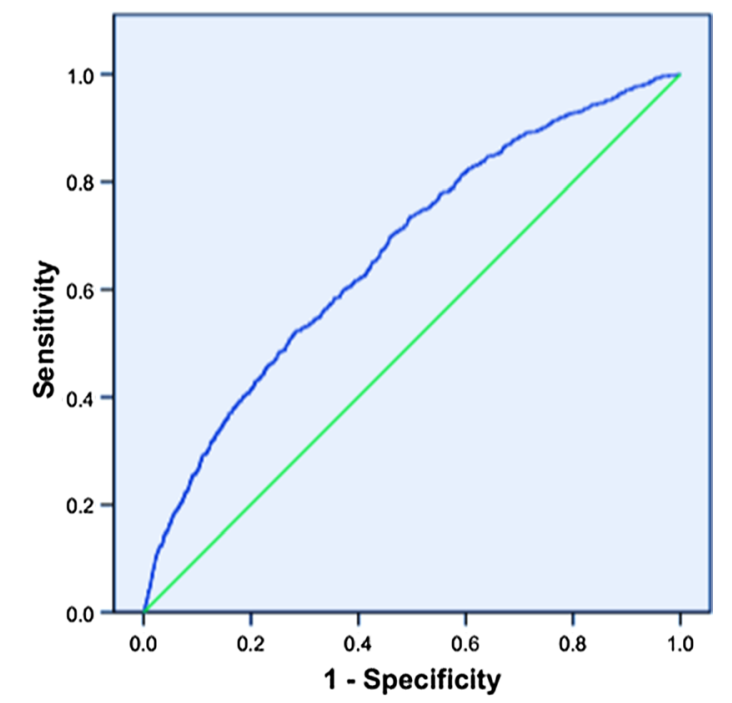

\begin{tabular}{lcccc} 
& AUC & Cut off value & Sensitivity (\%) & Specificity (\%) \\
\hline CVD & $0.663(0.645-0.681)$ & 9.03 & 59.23 & 63.15 \\
CHD & $0.669(0.651-0.688)$ & 9.03 & 59.96 & 62.84
\end{tabular}

CVD: cardiovascular disease; CHD: coronary heart disease; AUC: area under curve; TyG-index: triglyceride-glucose index

Fig. 2 Receiver operative characteristic curves and cut off values of TyG-index for incident CVD/CHD

36]. Moreover, we previously showed that the presence of IR among the non-hypertensive population was associated with CVD events [37]. There is a plausible biological mechanism underlying the association between TyGindex and incident CVD through the strong relationship between IR and endothelial dysfunction [38]. This mostly includes inflammation and functional impairment in the endothelium of blood vessels [39-41] leading to atherosclerosis and CVD [42]. However, the precise mechanism underlying the association between IR and CVD remains unclear. Therefore, considering the difficulty in direct estimation of insulin action and due to the absence of a standard insulin assay, TyG-index as a surrogate of IR may be associated with incident CVD.

\section{TyG-index and Cardiovascular Outcomes}

The association between TyG-index and CVD has also been supported by cross-sectional [43-48] and casecontrol studies $[49,50]$. Moreover, to the best of our knowledge, there have been three prospective [13-15] and three retrospective $[16,51,52]$ studies examining the association between TyG-index and cardiovascular outcomes among participants without baseline CVD.
Recently in a retrospective cohort study, Li et al. [16] evaluated the risk of TyG-index on incident CVD among the elderly population. Compared to the lowest quartile, the highest quartile was associated with a higher risk for CVD in the multivariate-adjusted model. Furthermore, Park et al. [51] reported that TyG-index is an independent predictor of coronary artery calcification progression. Moreover, in a study conducted among South Korean adults, TyG-index was reported as an independent predictor of coronary artery calcification (CAC) progression; this issue was more prominent among adults without heavy CAC at baseline [52].

Among non-obese and relatively healthy American white males, TyG-index $\geq 15$ was associated with higher risk of CVD/CHD mortality; however, the risk of TyGindex disappeared after further adjustment for non-HDLC [13]. Sánchez Iñigo et al. [14] reported that TyG-index was significantly associated with a higher risk for incident CVD i.e. those at the fourth and fifth quintiles had 1.52 and 2.32 higher risk for CVD events among the European population. Meanwhile Salazar et al. [15] conducted a study among Argentinians aged 15-80 years, reporting that TyG-index as a continuous variable was associated with $46 \%$ higher risk for CVD in multivariate analysis; 

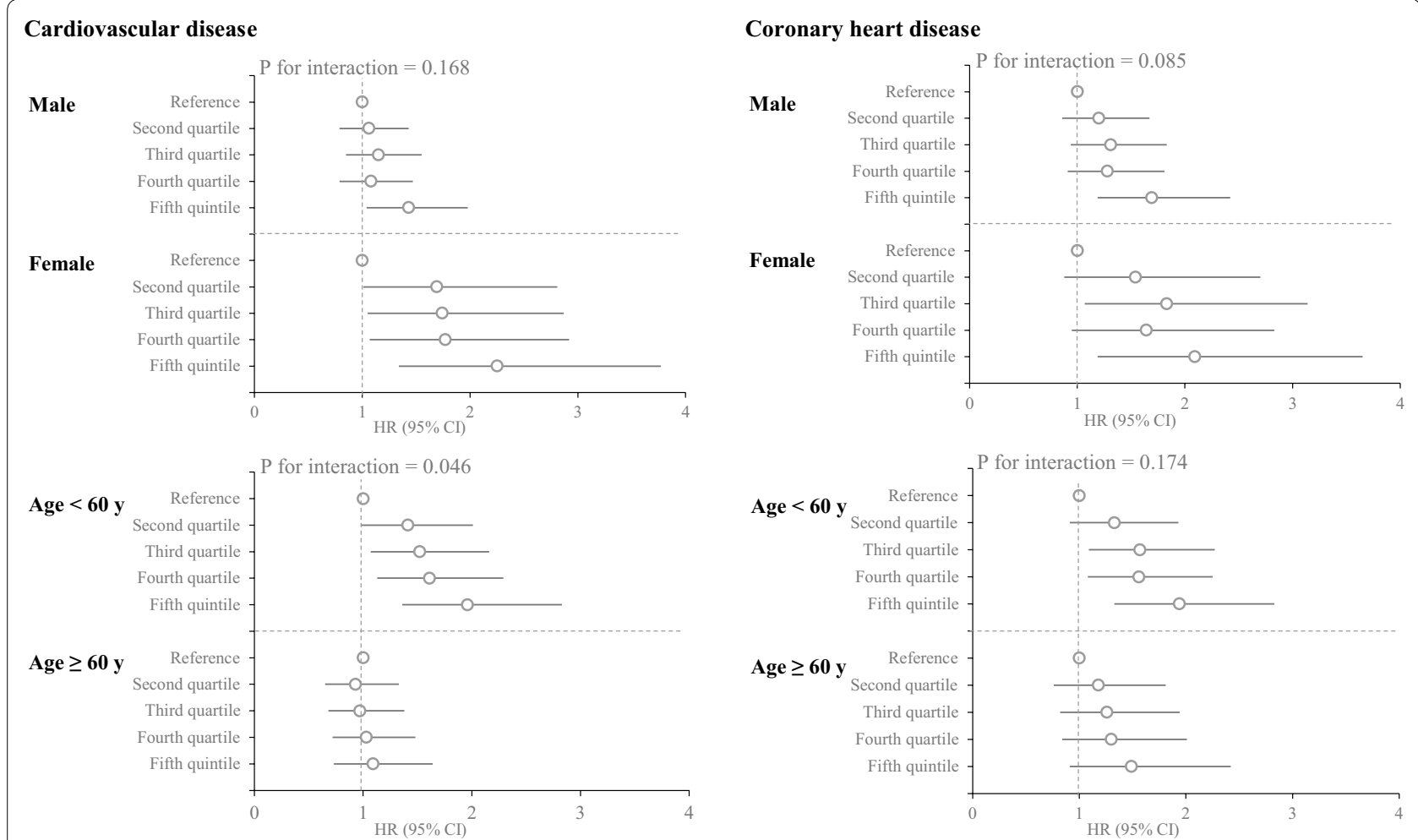

Fig. 3 Association between TyG-index quintiles and incident CVD/CHD stratified by gender and age. Stratified analyses were adjusted for WC, BMI, education, smoking status, physical activity, family history of CVD, T2D, hypertension, lowering lipid drugs, LDL-C, and HDL-C plus "age" in gender-stratified and "gender" in age-stratified analyses. TyG-index: Triglyceride-Glucose index; CVD: cardiovascular disease; CHD: coronary heart disease; WC: waist circumference; BMI: body mass index; T2D: type 2 diabetes; LDL-C: low density lipoprotein cholesterol; HDLC: high density lipoprotein cholesterol; HR: hazard ratio; Cl: confidence interval

Table 4 Clinical performance of the model to predict 10-year cardiovascular risk containing FRS and FRS + TyGindex models in the Population $<60$ years $(n=6175)$ : Tehran Lipid and Glucose Study

\begin{tabular}{ccccc}
\hline & $\begin{array}{l}\text { FRS model* } \\
\text { HR }(\mathbf{9 5 \%} \mathbf{~ C l})\end{array}$ & P-value & $\begin{array}{l}\text { FRS+TyG-index } \\
\text { HR (95\% Cl) }\end{array}$ & P-value \\
\hline Model components & & & \\
FRS $\quad 1.07(1.06-1.07)$ & $<0.001$ & $1.06(1.05-1.06)$ & $<0.001$ \\
TyG-index & - & & $1.42(1.26-1.60)$ & $<0.001$ \\
Model predictive performance indexes & & \\
C-index $\quad 0.789(0.773-0.805)$ & $<0.001$ & $0.771(0.752-$ & $<0.001$ \\
& & & $0.789)$ & \\
IDI & - & $0.001(-0.004$ to & 0.673 \\
& & & $0.007)$ & \\
\hline
\end{tabular}

FRS Framingham risk score, TyG-index Triglyceride-Glucose index, HR hazard ratio, Cl confidence interval, C-index Harrell's concordance statistic, IDI integrated discrimination improvement

*FRS model: Framingham risk score model including age, gender, total cholesterol, high density lipoprotein cholesterol, systolic blood pressure, antihypertensive drugs, smoking, and type 2 diabetes however, the risk for this measure did not reach a significant level when TyG-index was treated as a categorical variable. In our data analysis, the fifth quintile of TyGindex had about 60 and $80 \%$ higher risk for incident CVD and CHD in the multivariate analyses, respectively. We also found that a 1-SD increase in TyG-index was associated with about $20 \%$ higher risk for CVD events.

As expected, modifiable traditional risk factors including abdominal obesity, smoking, hypertension, T2D, LDL-C remained significantly associated with incident $\mathrm{CVD} /$ CHD; however, BMI and female gender were inversely associated with the incidence of both events in multivariate analysis. In the current study, in the absence of obesity mediators, BMI showed a significant risk for CVD events whereas together they were not associated with either event. Finally, when we adjusted WC in the analysis (an index of abdominal obesity), BMI changed direction, becoming inversely associated with incident CVD/ CHD. This finding might be due to the fact that BMI is a reflection of lean mass for individuals with the same $\mathrm{WC}$, whereas WC is a reflection of abdominal fat content for individuals with the same BMI [53]. Thus, we supposed 
that in our data analysis, BMI value when adjusted for WC might indicate the level of muscle mass. In line with our results, BMI was inversely associated with all-cause mortality in the Canadian population [54]. Regarding the lower risk of female gender, an issue addressed in our previous articles $[28,55]$, this might be related to baseline socioeconomic differences among genders. Our study was initiated in 1999-2000 in a developing country where females share in public sector employment was registered at 30.33 percent in 1999 and most females worked in households, whereas males were mostly occupied with outdoor jobs [56]. Hence, males were more in contact with chemicals and non-chemical environmental factors such as temperature, noise exposure, physiological stress, and air pollution which potentially amplify its association with cardiovascular events [57].

\section{TyG-index cut off point}

Considering TyG-index cut-off points, cohort studies conducted among Asian populations examined this issue for identifying the development of T2D. In a study conducted among Korean adults, the optimal cut-off value for TyG-index was 8.8, with the area under the ROC curve amounting to 0.751 (95\% CI 0.704-0.799) [58]. Another study conducted in Korea reported a cutoff point of 8.86 for TyG-index (sensitivity of 56.01 and specificity of $57.11 \%$ ) for predicting T2D among males, with the corresponding value for females being 8.52 (sensitivity of 67.25 and specificity of $55.85 \%$ ) [59]. The cut-off values of TyG-index for prevalent hypertension in a cross-sectional study among the Chinese population were reported as 9.04 (sensitivity of 52.58 and specificity of $72.27 \%$ ) in males and 8.59 (sensitivity of 76.07 and specificity of $46.69 \%$ ) in females [60]. Mao et al. estimated a cut-off value of 8.56 with sensitivity of 87.2 and specificity of $35.3 \%$ for TyG-index for recurrent cardiovascular outcomes, which was assessed by ROC curve analysis [61]. Hence, our derived corresponding cut-off points of TyG-index for incident CVD and CHD among Iranian population were comparable to those values for detecting prevalent hypertension and were higher than those reported for predicting incident T2D and recurrent CVD among the East Asian population.

\section{Added value of TyG-index to FRS}

We previously validated and examined the clinical usefulness of FRS in prediction of CVD events among Tehranian population (30-74 years) [19]. In the current study, we fitted predictive models for incident CVD among younger adults using FRS variables, with and without further addition of the TyG-index. In our data analyses, no improvement was observed in the prediction of CVD risk after further addition of the TyG-index to the Framingham predictors. In this regard, Sanchez et al. [14] plotted AUC of the ROC curves for the Framingham model and Framingham + TyG-index model. The added predictive value of the model including TyG-index was seen only among participants at 10 to $20 \%$ of 10 -year cardiovascular risk.

\section{Strengths and limitations}

There are some strengths for this study that should be mentioned. This is the first long-term population-based prospective study conducted in the MENA region in a population with a high burden of CVD events. Second, we assessed the precise value of CVD risk factors rather than relying on self-reported data. Third, the statistical power of our study according to the effect size of the fifth quintile and a 1-SD increase TyG-index in the multivariate analysis were $96 \%$ and $33 \%$, respectively.

There are a few limitations that must also be noted. First, previous studies reported that variation of TyGindex during the follow-up time could modify the relationship between this index and CVD [61]. We used only the baseline values of TyG-index and other risk factors and we did not consider changes in these parameters over the follow-up time. Second, this is a population-based study conducted among participants resident in the metropolitan city of Tehran; hence, our findings may not be generalizable to other populations. Third, we did not record nutritional habits or energy intake which might affected TG levels. However, we did adjust for other related confounders such as BMI and lipid measures.

\section{Conclusion}

During more than a decade of follow-up we found that TyG-index (as a surrogate for IR) was a significant risk factor for incident CVD/CHD, whether as a continuous or categorical variable; an issue that was more prominent among the younger population. In addition, we recommend a cut-off point of 9 for TyG-index to identify Iranian adults who are at risk for future CVD/ CHD. Finally, adding TyG-index to FRS does not provide greater risk prediction for CVD.

\section{Supplementary information}

Supplementary information accompanies this paper at https://doi. org/10.1186/s12933-020-01121-5.

Additional file 1: Table S1. Adjusted hazard ratio of BMI for incident CVD according to Cox proportional hazard models: Tehran Lipid and Glucose Study. Tables S2. Adjusted hazard ratio of BMI for incident CHD according to Cox proportional hazard models: Tehran Lipid and Glucose Study.

\section{Abbreviations}

CVD: Cardiovascular diseases; MENA: Middle East and North Africa; IR: Insulin resistance; T2D: Type 2 diabetes; TyG-index: Triglyceride-glucose index; TG:

Triglyceride; FPG: Fasting plasma glucose; CHD: Coronary heart disease; TLGS Tehran Lipid and Glucose Study; LRC: Lipid Research Clinic; MAQ: Modifiable 
Activity Questionnaire; BMI: Body mass index; SBP: Systolic blood pressure; DBP: Diastolic blood pressure; TC: Total cholesterol; HDL-C: High-density lipoprotein cholesterol; 2hPG: 2 h plasma glucose; LDL-C: Low-density lipoprotein cholesterol; MI: Myocardial infarction; ECG: Electrocardiogram; MET: Metabolic equivalent task; SD: Standard deviation; HR: Hazard ratio; Cl: Confidence interval; WC: Waist circumference; ROC: Receiver operation characteristic.

\section{Acknowledgements}

We express our appreciation to the research team members and to TLGS participants for their contribution to the study.

\section{Authors' contributions}

$\mathrm{NB}, \mathrm{MT}$ and $\mathrm{FH}$ raised the presented idea and designed the study. MH and NB conducted the analyses. $\mathrm{FH}, \mathrm{MT}, \mathrm{NB}$, and $\mathrm{MH}$ interpreted the results. NB and MT developed the first draft of the manuscript. FH and FA critically reviewed the manuscript. All authors read and approved the final manuscript.

\section{Funding}

This study was supported in part by Grant No 23160-7 from Shahid Beheshti University of Medical Sciences.

\section{Availability of data and materials}

The datasets used and/or analyzed during the current study are available from the corresponding author on reasonable request.

\section{Ethics approval and consent to participate}

The research protocol was approved by the ethics committee of the Research Institute for Endocrine Sciences, Shahid Beheshti University of Medical Sciences, Tehran, Iran.

\section{Consent for publication}

Not applicable.

\section{Competing interests}

The authors declare that they have no competing interests.

\section{Author details}

${ }^{1}$ Prevention of Metabolic Disorders Research Center, Research Institute for Endocrine Sciences, Shahid Beheshti University of Medical Sciences, No. 24 Aarabi St. Velenjak, P.O. Box: 19395-4763, Tehran, Iran. ${ }^{2}$ Endocrine Research Center, Research Institute for Endocrine Sciences, Shahid Beheshti University of Medical Sciences, Tehran, Iran.

Received: 13 May 2020 Accepted: 12 September 2020

Published online: 29 September 2020

\section{References}

1. Roth GA, Abate D, Abate KH, Abay SM, Abbafati C, et al. Global, regional, and national age-sex-specific mortality for 282 causes of death in 195 countries and territories, 1980-2017: a systematic analysis for the Global Burden of Disease Study 2017. Lancet. 2018;392:1736-88,

2. Turk-Adawi K, Sarrafzadegan N, Fadhil I, Taubert K, Sadeghi M, et al. Cardiovascular disease in the Eastern Mediterranean region: epidemiology and risk factor burden. Nat Rev Cardiol. 2018;15:106.

3. Mensah GA, Roth GA, Fuster V. The Global Burden of Cardiovascular Diseases and Risk Factors: 2020 and Beyond. J Am Coll Cardiol. 2019;74(20):2529-32.

4. Ginsberg HN. Insulin resistance and cardiovascular disease. J Clin Investig. 2000;106:453-8.

5. Jeppesen J, Hansen TW, Rasmussen S, Ibsen H, Torp-Pedersen C, et al. Insulin resistance, the metabolic syndrome, and risk of incident cardiovascular disease: a population-based study. J Am Coll Cardiol. 2007:49:2112-9.

6. Simental-Mendía LE, Rodríguez-Morán M, Guerrero-Romero F. The product of fasting glucose and triglycerides as surrogate for identifying insulin resistance in apparently healthy subjects. Metab Syndr Related Disord. 2008;6:299-304.

7. Abbasi F, Reaven GM. Comparison of two methods using plasma triglyceride concentration as a surrogate estimate of insulin action in nondiabetic subjects: triglycerides $\times$ glucose versus triglyceride/highdensity lipoprotein cholesterol. Metabolism. 2011;60:1673-6.

8. Toro-Huamanchumo CJ, Urrunaga-Pastor D, Guarnizo-Poma M, LazaroAlcantara H, Paico-Palacios S, et al. Triglycerides and glucose index as an insulin resistance marker in a sample of healthy adults. Diabetes Metab Syndr. 2019;13:272-7.

9. Lee $\mathrm{S}-\mathrm{H}$, Kwon H-S, Park Y-M, Ha H-S, Jeong SH, et al. Predicting the development of diabetes using the product of triglycerides and glucose: the Chungju Metabolic Disease Cohort (CMC) study. PLoS ONE. 2014;9:e90430.

10. Da Young Lee ESL, Kim JH, Park SE, Park C-Y, Oh K-W, et al. Predictive value of triglyceride glucose index for the risk of incident diabetes: a 4-year retrospective longitudinal study. PLOS ONE. 2016;11(9):e0163465.

11. Tohidi M, Baghbani-Oskouei A, Ahanchi NS, Azizi F, Hadaegh F. Fasting plasma glucose is a stronger predictor of diabetes than triglycerideglucose index, triglycerides/high-density lipoprotein cholesterol, and homeostasis model assessment of insulin resistance: Tehran Lipid and Glucose Study. Acta Diabetol. 2018;55:1067-74.

12. Chamroonkiadtikun P, Ananchaisarp T, Wanichanon W. The triglycerideglucose index, a predictor of type 2 diabetes development: a retrospective cohort study. Primary Care Diabetes. 2020;14:161-7.

13. Vega GL, Barlow CE, Grundy SM, Leonard D, DeFina LF. Triglyceride-tohigh-density-lipoprotein-cholesterol ratio is an index of heart disease mortality and of incidence of type 2 diabetes mellitus in men. J Investig Med. 2014;62:345-9.

14. Sánchez-Iñigo L, Navarro-González D, Fernández-Montero A, PastranaDelgado J, Martínez JA. The TyG index may predict the development of cardiovascular events. Eur J Clin Invest. 2016;46:189-97.

15. Salazar M, Carbajal H, Espeche W, Aizpurúa M, Dulbecco C, et al. Comparison of two surrogate estimates of insulin resistance to predict cardiovascular disease in apparently healthy individuals. Nutr Metab Cardiovasc Dis. 2017;27:366-73.

16. Li S, Guo B, Chen H, Shi Z, Li Y, et al. The role of the triglyceride (triacylglycerol) glucose index in the development of cardiovascular events: a retrospective cohort analysis. Sci Rep. 2019;9:7320.

17. Luo E, Wang D, Yan G, Qiao Y, Liu B, et al. High triglyceride-glucose index is associated with poor prognosis in patients with acute ST-elevation myocardial infarction after percutaneous coronary intervention. Cardiovasc Diabetol. 2019;18:150.

18. Derakhshan A, Tohidi M, Hajebrahimi M, Saadat N, Azizi F, et al. Sexspecific incidence rates and risk factors of insulin resistance and $\beta$-cell dysfunction: a decade follow-up in a Middle Eastern population. Diabet Med. 2017;34:245-52.

19. Khalili D, Hadaegh F, Soori H, Steyerberg EW, Bozorgmanesh M, et al. Clinical usefulness of the Framingham cardiovascular risk profile beyond its statistical performance: the Tehran Lipid and Glucose Study. Am J Epidemiol. 2012;176:177-86.

20. Azizi F, Ghanbarian A, Momenan AA, Hadaegh F, Mirmiran P, et al. Prevention of non-communicable disease in a population in nutrition transition: Tehran Lipid and Glucose Study phase II. Trials. 2009;10:5.

21. Ainsworth BE, Jacobs JD, Leon AS. Validity and reliability of self-reported physical activity status: the Lipid Research Clinics questionnaire. Med Sci Sports Exerc. 1993;25:92-8.

22. Kriska AM, Knowler WC, LaPorte RE, Drash AL, Wing RR, et al. Development of questionnaire to examine relationship of physical activity and diabetes in Pima Indians. Diabetes Care. 1990;13:401-11.

23. Kheirandish M, Asgari S, Lotfaliany M, Bozorgmanesh M, Saadat N, et al. Secular trends in serum lipid levels of a Middle Eastern adult population; 10 years follow up in Tehran lipid and glucose study. Lipids Health Dis. 2014;13:20.

24. Chen Y, Zhang X, Pan B, Jin X, Yao H, et al. A modified formula for calculating low-density lipoprotein cholesterol values. Lipids Health Dis. 2010;9:52.

25. Hadaegh F, Harati H, Ghanbarian A, Azizi F. Association of total cholesterol versus other serum lipid parameters with the short-term prediction of cardiovascular outcomes: Tehran Lipid and Glucose Study. Eur J Cardiovasc Prev Rehab. 2006;13:571-7.

26. Guerrero-Romero F, Simental-Mendía LE, Gonzalez-Ortiz M, MartínezAbundis E, Ramos-Zavala MG, et al. The product of triglycerides and glucose, a simple measure of insulin sensitivity. Comparison with 
the euglycemic-hyperinsulinemic clamp. J Clin Endocrinol Metab. 2010;95:3347-51.

27. Committee IR (2005) Guidelines for data processing and analysis of the International Physical Activity Questionnaire (IPAQ)-short and long forms. http://www.ipaqkise/scoringpdf.

28. Sardarinia M, Akbarpour S, Lotfaliany M, Bagherzadeh-Khiabani F, Bozorgmanesh M, et al. Risk factors for incidence of cardiovascular diseases and all-cause mortality in a middle eastern population over a decade followup: Tehran lipid and glucose study. PLoS ONE. 2016;11:e0167623.

29. Belsley DA, Kuh E, Welsch RE. Regression diagnostics: identifying influential data and sources of collinearity. Hoboken: Wiley; 2005.

30. Harrell FE, Califf RM, Pryor DB, Lee KL, Rosati RA. Evaluating the yield of medical tests. JAMA. 1982;247:2543-6.

31. Hlatky MA, Greenland P, Arnett DK, Ballantyne CM, Criqui MH, et al. Criteria for evaluation of novel markers of cardiovascular risk: a scientific statement from the American Heart Association. Circulation. 2009; 119:2408-16.

32. Lu Y, Hajifathalian K, Ezzati M, Woodward M, Rimm EB, et al. Metabolic mediators of the effects of body-mass index, overweight, and obesity on coronary heart disease and stroke: a pooled analysis of 97 prospective cohorts with 1. 8 million participants. Lancet. 2013;383:970-83.

33. Warram JH, Martin BC, Krolewski AS, Soeldner JS, Kahn CR. Slow glucose removal rate and hyperinsulinemia precede the development of type II diabetes in the offspring of diabetic parents. Ann Intern Med. 1990;113:909-15.

34. Wang F, Han L, Hu D. Fasting insulin, insulin resistance and risk of hypertension in the general population: a meta-analysis. Clin Chim Acta. 2017:464:57-63.

35. Gast KB, Tjeerdema N, Stijnen T, Smit JW, Dekkers OM. Insulin resistance and risk of incident cardiovascular events in adults without diabetes: meta-analysis. PLoS ONE. 2012;7:e52036.

36. Laakso M, Kuusisto J. Insulin resistance and hyperglycaemia in cardiovascular disease development. Nat Rev Endocrinol. 2014;10:293-302.

37. Ghoreishian H, Tohidi M, Derakhshan A, Hajsheikholeslami F, Azizi F, et al. Presence of hypertension modifies the impact of insulin resistance on incident cardiovascular disease in a Middle Eastern population: the Tehran Lipid and Glucose Study. Diabet Med. 2015;32:1311-8.

38. Janus A, Szahidewicz-Krupska E, Mazur G, Doroszko A. Insulin resistance and endothelial dysfunction constitute a common therapeutic target in cardiometabolic disorders. Mediators Inflamm. 2016:2016:3634948.

39. Festa A, Hanley AJ, Tracy RP, D'Agostino R Jr, Haffner SM. Inflammation in the prediabetic state is related to increased insulin resistance rather than decreased insulin secretion. Circulation. 2003;108:1822-30.

40. Vincent MA, Montagnani M, Quon MJ. Molecular and physiologic actions of insulin related to production of nitric oxide in vascular endothelium. Curr DiabRep. 2003;3:279-88.

41. Montagnani M, Golovchenko I, Kim I, Koh GY, Goalstone ML, et al. Inhibition of phosphatidylinositol 3-kinase enhances mitogenic actions of insulin in endothelial cells. J Biol Chem. 2002;277:1794-9.

42. Kim J-a, Montagnani M, Koh KK, Quon MJ. Reciprocal relationships between insulin resistance and endothelial dysfunction: molecular and pathophysiological mechanisms. Circulation. 2006;113:1888-904.

43. Kim MK, Ahn CW, Kang S, Nam JS, Kim KR, et al. Relationship between the triglyceride glucose index and coronary artery calcification in Korean adults. Cardiovasc Diabetol. 2017:16:108.

44. Lambrinoudaki I, Kazani MV, Armeni E, Georgiopoulos G, Tampakis K, et al. The TyG index as a marker of subclinical atherosclerosis and arterial stiffness in lean and overweight postmenopausal women. Heart Lung Circ. 2018;27:716-24

45. Won K-B, Park G-M, Lee S-E, Cho I-J, Kim HC, et al. Relationship of insulin resistance estimated by triglyceride glucose index to arterial stiffness. Lipids Health Dis. 2018;17:268.
46. da Silva A, Caldas APS, Hermsdorff HHM, Bersch-Ferreira ÂC, Torreglosa $C R$, et al. Triglyceride-glucose index is associated with symptomatic coronary artery disease in patients in secondary care. Cardiovasc Diabetol. 2019;18:89.

47. Zhao S, Yu S, Chi C, Fan X, Tang J, et al. Association between macro-and microvascular damage and the triglyceride glucose index in communitydwelling elderly individuals: the Northern Shanghai Study. Cardiovasc Diabetol. 2019;18:95.

48. Morales-Gurrola G, Simental-Mendía L, Castellanos-Juárez F, SalasPacheco J, Guerrero-Romero F. The triglycerides and glucose index is associated with cardiovascular risk factors in metabolically obese normalweight subjects. J Endocrinol Investig. 2020; 1-6.

49. Jin J-L, Cao Y-X, Wu L-G, You X-D, Guo Y-L, et al. Triglyceride glucose index for predicting cardiovascular outcomes in patients with coronary artery disease. J Thorac Dis. 2018;10:6137.

50. Jin J-L, Sun D, Cao Y-X, Guo Y-L, Wu N-Q, et al. Triglyceride glucose and haemoglobin glycation index for predicting outcomes in diabetes patients with new-onset, stable coronary artery disease: a nested casecontrol study. Ann Med. 2018;50:576-86.

51. Park K, Ahn CW, Lee SB, Kang S, Nam JS, et al. Elevated TyG Index Predicts Progression of Coronary Artery Calcification. Diabetes Care. 2019:42:1569-73.

52. Won K-B, Park EJ, Han D, Lee JH, Choi S-Y, et al. Triglyceride glucose index is an independent predictor for the progression of coronary artery calcification in the absence of heavy coronary artery calcification at baseline. Cardiovasc Diabetol. 2020;19:1-8.

53. Bigaard J, Tjønneland A, Thomsen BL, Overvad K, Heitmann BL, et al. Waist circumference, BMI, smoking, and mortality in middle-aged men and women. Obes Res. 2003;11:895-903.

54. Janssen I, Katzmarzyk PT, Ross R. Body mass index is inversely related to mortality in older people after adjustment for waist circumference. J Am Geriatr Soc. 2005;53:2112-8.

55. Khalili D, Sheikholeslami FH, Bakhtiyari M, Azizi F, Momenan AA, et al. The incidence of coronary heart disease and the population attributable fraction of its risk factors in Tehran: a 10-year population-based cohort study. PLoS ONE. 2014;9:e105804.

56. Alaedini P, Razavi MR. Women's participation and employment in Iran: a critical examination. Critique. 2005;14:57-73.

57. Rajagopalan S, Al-Kindi SG, Brook RD. Air pollution and cardiovascular disease: JACC state-of-the-art review. J Am Coll Cardiol. 2018;72:2054-70.

58. Lee ES, Kim JH, Park SE, Park C-Y, Oh K-W, et al. Predictive value of triglyceride glucose index for the risk of incident diabetes: a 4-year retrospective longitudinal study. PLoS ONE. 2016;11:e0163465.

59. Lee J-W, Lim N-K, Park H-Y. The product of fasting plasma glucose and triglycerides improves risk prediction of type 2 diabetes in middle-aged Koreans. BMC Endocr Disord. 2018;18:33.

60. Jian S, Su-Mei N, Xue C, Jie Z, Xue-sen W. Association and interaction between triglyceride-glucose index and obesity on risk of hypertension in middle-aged and elderly adults. Clin Exp Hypertens. 2017;39:732-9.

61. Mao Q, Zhou D, Li Y, Wang Y, Xu S-C, et al. (2019) The triglyceride-glucose index predicts coronary artery disease severity and cardiovascular outcomes in patients with non-st-segment elevation acute coronary syndrome. Dis Markers 2019.

\section{Publisher's Note}

Springer Nature remains neutral with regard to jurisdictional claims in published maps and institutional affiliations. 